\title{
IDEALS OF FREE INVERSE SEMIGROUPS
}

\author{
W. D. MUNN \\ (Received 27 February 1980) \\ Communicated by T. E. Hall
}

\begin{abstract}
It is shown that no proper ideal of a free inverse semigroup is free and that every isomorphism between ideals is induced by a unique autonorphism of the whole semigroup. In addition, necessary and sufficient conditions are given for two principal ideals to be isomorphic.
\end{abstract}

1980 Mathematics subject classificution (Amer. Math. Soc.): 20 M 99.

This paper is concerned with various properties of (two-sided) ideals of a free inverse semigroup $S$, the investigation being based on Scheiblich's structure theorem for $S$ (Scheiblich (1972), (1973)).

We show first that no proper ideal of $S$ is a free inverse subsemigroup of $S$ (Theorem 1). A complementary result, stating that every ideal of $S$ contains an isomorphic copy of $S$, is a consequence of a theorem established by the author in a separate note (Munn (to appear)).

The second topic discussed is that of isomorphisms between ideals of $S$. Every isomorphism from one ideal of $S$ to another can be extended to a unique automorphism of $S$ (Theorem 2): consequently, the automorphism group of an ideal of $S$ is a subgroup of the automorphism group of $S$ itself. A description of the automorphism group of $S$ has been provided by O'Carroll (1974).

Necessary and sufficient conditions are given for two principal ideals of $S$ to be isomorphic (Theorem 3 ) and for the inverse subsemigroups of $S$ generated by two $\mathscr{f}$ classes to be isomorphic (Theorem 4). Theorems 3 and 4 answer two questions raised by Reilly (1976).

The notation and terminology throughout will be that of Howie (1976).

Let $S$ be an inverse semigroup. The semilattice of $S$ and the automorphism group of $S$ will be denoted by $E(S)$ and aut $S$ respectively. Also, if $A$ is a nonempty subset of $S$ then $A^{-1}$ denotes $\left\{a^{-1}: a \in A\right\}$ and so the inverse subsemigroup of $S$ generated by $A$ is just $\left\langle A \cup A^{-1}\right\rangle$, the subsemigroup generated by $A \cup A^{-1}$. 
The following easily-checked facts will be used below without subsequent comment. If $V$ is an inverse subsemigroup of $S$ then any $\mathscr{R}$-class [ $\mathscr{f}$-class] of $S$ contained in $V$ is also an $\mathscr{R}$-class [ $\mathscr{J}$-class] of $V$. Moreover, if $T$ and $U$ are inverse subsemigroups of $S$ and $t \in T$ is such that $R_{t} \subseteq T$ then, for any isomorphism $\theta: T \rightarrow U$ for which $R_{t \theta} \subseteq U$, we have that $R_{t} \theta=R_{t \theta}$.

For the remainder of the paper $X$ will denote a nonempty set. Let $G=\mathscr{F}^{\mathscr{G}} \mathscr{G}_{X}$, the free group on $X$, and let 1 denote the identity of $G$. The length $\|(a)$ of $a \in G$ is defined by

$$
\nVdash(a)= \begin{cases}n & \text { if the reduced form of } a \text { is } x_{1} x_{2} \ldots x_{n} \\ 0 & \text { if } a=1 .\end{cases}
$$

For all $a \in G$ the set of all initial segments (including 1) of the reduced form of $a$ will be denoted by $\bar{a}$; further, for all nonempty subsets $A$ of $G$ we write

$$
\bar{A}=\{\bar{a}: a \in A\}
$$

and we say that $A$ is left closed if and only if $\bar{A}=A$.

We now describe the construction for the free inverse semigroup on $X$ given by Scheiblich ((1972), (1973)). Let $\mathscr{Y}$ denote the set of all finite left closed subsets of $G$ with at least two elements. Write

$$
S=\{(A, g) \in \mathscr{Y} \times G: g \in A\} .
$$

It is readily verified that if $(A, g)$ and $(B, h)$ are in $S$ then $A \cup g B \in \mathscr{Y}$; hence we can define a multiplication on $S$ by the rule that

$$
(A, g)(B, h)=(A \cup g B, g h) .
$$

With respect to this multiplication $S$ is an inverse semigroup in which

$$
(\forall(A, g) \in S), \quad(A, g)^{-1}=\left(g^{-1} A, g^{-1}\right)
$$

and

$$
E(S)=\{(A, 1): A \in \mathscr{Y}\}
$$

Let us write

$$
W=\{(\bar{x}, x): x \in X\} .
$$

(Note that, for all $x \in X, \bar{x}=\{1, x\}$.) Then $S=\left\langle W \cup W^{-1}\right\rangle$ and each mapping from $W$ to an inverse semigroup $T$ extends to a unique homomorphism from $S$ to $T$. Accordingly, $S$ is the free inverse semigroup on $X$ and $W$ is a set of free generators of $S$ (see Reilly (1972), (1973)). We denote $S$, as constructed above, by $\mathscr{F}_{\mathscr{I}_{X}}$. The cardinal $|X|$ of $X$ is termed the rank of $S$.

The mapping $\pi: S \rightarrow G$ defined by

$$
(A, g) \pi=g
$$

is evidently a surjective homomorphism. Now suppose that $T$ is an inverse 
subsemigroup of $S$. It is straightforward to prove that

$$
(\forall a, b \in T), \quad a \pi=b \pi \Leftrightarrow e a=e b \quad \text { for some } e \in E(T) .
$$

Thus $\pi$ induces the least group congruence on $T$ (Munn (1961)). Moreover, if $T$ is an ideal of $S$ then $T \pi=G$.

It will be convenient to denote the $\mathscr{Y}$-component of a typical element a of $S$ by $\mathscr{S}(a)$. Thus

$$
(\forall a \in S), \quad a=(\mathscr{P}(a), a \pi) .
$$

Green's relations on $S$ are characterized in Reilly (1972) and further properties of $S$ are listed in Reilly (1976). We note, in particular, that $S$ is combinatorial and completely semisimple and that its partially ordered set of $\mathscr{J}$-classes satisfies the maximal condition. Since elements $a$ and $b$ of $S$ are $\mathscr{R}$-equivalent if and only if $\mathscr{P}(a)=\mathscr{P}(b)$, we have that

$$
(\forall a \in S), \quad R_{a} \pi=\mathscr{P}(a) .
$$

Each $\mathscr{J}$-class of $S$ is finite: specifically,

$$
(\forall a \in S), \quad\left|J_{a}\right|=|\mathscr{S}(a)|^{2} .
$$

Reilly (1972) has shown that every set of free generators of $S$ is contained in $W \cup W^{-1}$. The following property therefore follows from (P3).

$$
\text { If } a \text { belongs to a set of free generators of } S \text { then }\left|J_{a}\right|=4 \text {. }
$$

For ease of reference we also record that

$$
(\forall a, b \in S), \quad(a, b) \in \mathscr{J} \Leftrightarrow \mathscr{S}(a)=g^{-1} \mathscr{S}(b) \quad \text { for some } g \in \mathscr{P}(b) .
$$

Unlike the corresponding situation for free groups, not every inverse subsemigroup of a free inverse semigroup $S$ is free; for example, $E(S)$ is not a free inverse semigroup. By a proper idcal of $S$ we mean an ideal other than $S$ itself. We now establish

THEOREM 1. No proper ideal of a free inverse semigroup $S$ is a free inverse subsemigroup of $S$.

Proof. Take $S=\mathscr{F} \mathscr{I}_{\boldsymbol{X}}$. Suppose that $M$ is an ideal of $S$ which is also a free inverse subsemigroup of $S$. We shall show that $M=S$.

Let $K$ be a set of free generators of $M$ and let $a \in K$. Then, by (P4) (with $M$ replacing $S$ ), the $\mathscr{J}$-class of $M$ containing $a$ has exactly 4 elements. Since $M$ is an ideal of $S$ this means that $\left|J_{a}\right|=4$. Consequently, by (P3), $|\mathscr{P}(a)|=2$ and so, since $a^{2} \neq a$, we have that $a \in W \cup W^{-1}$. Thus $K \subseteq W \cup W^{-1}$ and therefore $K \cup K^{-1} \subseteq W \cup W^{-1}$. Hence $\left(K \cup K^{-1}\right) \pi \subseteq\left(W \cup W^{-1}\right) \pi=X \cup X^{-1}$. 
Now suppose that there exists $b \in\left(W \cup W^{-1}\right) \backslash\left(K \cup K^{-1}\right)$. Then $b \pi \in\left(X \cup X^{-1}\right) \backslash\left(K \cup K^{-1}\right) \pi$, since $\left.\pi\right|_{\boldsymbol{W} \cup \boldsymbol{W}^{-1}}$ is injective. Let $e \in E(M)$. Then $e b \in M$ and so there exist elements $k_{1}, k_{2}, \ldots, k_{n}$ in $K \cup K^{-1}$ such that

$$
k_{1} k_{2} \ldots k_{n}=e b \text {. }
$$

Since $e \pi=1$ it follows that

$$
\left(k_{1} \pi\right)\left(k_{2} \pi\right) \ldots\left(k_{n} \pi\right)(b \pi)^{-1}=1 .
$$

But

$$
k_{i} \pi \in\left(K \cup K^{-1}\right) \pi \subseteq X \cup X^{-1} \backslash\left\{b \pi,(b \pi)^{-1}\right\} \quad(i=1,2, \ldots, n)
$$

and so we have a contradiction. Thus $K \cup K^{-1}=W \cup W^{-1}$. Hence $M=\left\langle K \cup K^{-1}\right\rangle=\left\langle W \cup W^{-1}\right\rangle=S$ and the proof is complete.

Remark. Although a proper ideal of a free inverse semigroup $S$ is not itself a free inverse semigroup, it contains an isomorphic copy of $S$ (Munn (to appear) Remark 3).

We now examine isomorphisms between ideals of a free inverse semigroup. To save repetition we shall assume that $S=\mathscr{F} \mathscr{I}_{X}$ and $G=\mathscr{F} \mathscr{G}_{X}$ in the three lemmas below.

LEMma 1. Let $T$ and $U$ be inverse subsemigroups of $S$ and let $\theta: T \rightarrow U$ be an isomorphism. Then there exists an isomorphism $\phi: T \pi \rightarrow U \pi$ such that, for all $a \in T$, $a \pi \phi=a 0 \pi$. Suppose, further, that $t \in T$ is such that $R_{t} \subseteq T$ and $R_{t \theta} \subseteq U$. Then

$$
\left(\forall a \in R_{t}\right), \quad a \theta=(\mathscr{S}(a) \varphi, a \pi \varphi) \text {. }
$$

(Note that if $a \in R_{t}$ then $\mathscr{S}(a) \subseteq T \pi$, since $\mathscr{S}(a)=R_{t} \pi . h y(\mathrm{P} 2)$.)

Proof. Let $a, b \in T$ be such that $a \pi=b \pi$. Then, by (Pl), there exists $c^{\prime} \in E(T)$ such that $e a=e b$ and so $e \theta a \theta=e \theta b \theta$. Since $e \theta \in E(U)$, this shows that $a \theta \pi=b \theta \pi$. Thus we can define a mapping $\varphi: T \pi \rightarrow U \pi$ by the rule that

$$
(\forall a \in T), \quad a \pi \varphi=a(\theta \pi .
$$

Since $\theta$ is surjective, $\varphi$ is surjective. Now suppose that $a, b \in T$ are such that $a \pi \phi=b \pi \varphi$. Then, by (P1), there exists $f \in E(U)$ such that $f(a \theta)=f(b \theta)$ and so (ea) $\theta=(e b) \theta$, where $e=f \theta^{-1} \in E(T)$. Thus, since $\theta$ is injective, $e a=e b$. Consequently, by $(\mathrm{P} 1), a \pi=b \pi$. Hence $\varphi$ is injective. Since $\theta$ and $\pi$ are homomorphisms, so also is $\varphi$. Thus $\varphi$ is an isomorphism. 
Next, let $a \in R_{t}$. Since $R_{t} \subseteq T$ and $R_{t \theta} \subseteq U$ it follows that $R_{a} \theta=R_{t} \theta=R_{t \theta}=R_{a \theta}$ and so

$$
\begin{aligned}
a(\theta) & =(\mathscr{S}(a \theta), a \theta \pi) \\
& =\left(R_{a \theta} \pi, a \theta \pi\right) \quad \text { by }(\mathrm{P} 2) \\
& =\left(R_{a} \theta \pi, a \theta \pi\right) \\
& =\left(R_{a} \pi \varphi, a \pi \varphi\right) \quad \text { by }(1) \\
& =(\mathscr{S}(a) \varphi, a \pi \varphi) \quad \text { by }(\mathrm{P} 2) .
\end{aligned}
$$

This completes the proof.

Definition. An automorphism $\varphi$ of $G=\mathscr{F} \mathscr{G}_{X}$ is special if and only if $\left(X \cup X^{-1}\right) \varphi=X \cup X^{-1}$.

Let the set of all special automorphisms of $G$ be denoted by aut* $G$. It is clear that aut $G$ is a subgroup of aut $G$. If $X$ is finite with exactly $n$ elements then $\mid$ aut $^{*} G \mid=2^{n} n$ !.

Theorem 2 of O'Carroll (1974) shows, in effect, that

$$
\text { aut } S \cong \text { aut* } G \text {. }
$$

Lemma 2. Let $M$ and $N$ he ideals of $S$ and let $\theta: M \rightarrow N$ be an isomorphism. Then there exists $\varphi \in$ aut ${ }^{*} G$ such that

$$
(\forall a \in M), \quad a \theta=(\mathscr{P}(a) \varphi, a \pi \varphi) .
$$

Proof. Since $M$ and $N$ are ideals of $S$, each is a union of $\mathscr{R}$-classes of $S$. Also $M \pi=G=N \pi$. Hence, by L.emma 1 , there exists $\varphi \in$ aut $G$ such that

$$
(\forall a \in M), \quad a \theta=(\mathscr{S}(a) \varphi, a \pi \varphi) .
$$

It remains to show that $\varphi$ is special.

Suppose that there exists $x \in X \cup X^{-1}$ such that $l(x \varphi)>1$. Let $e \in E(M)$ and let $k=|\mathscr{S}(e)|$. Since $\mathscr{S}(e)$ is finite and left closed there exists a nonnegative integer $r$ such that $x^{n} \in \mathscr{P}(e)$ if $0 \leqslant n \leqslant r$ and $x^{n} \notin \mathscr{S}(e)$ if $n>r$. (By convention, $x^{0}=1$.) Let us write $f=e g$, where $g=\left(\overline{x^{r}-2 k}, 1\right)$. Then $f \in M$, since $M$ is an ideal of $S$. Now let $A=\left\{x^{r+1}, x^{r+2} \ldots, x^{r+2 k}\right\}$. We have that

$$
\mathscr{P}\left(f^{\prime}\right)=\mathscr{S}(e) \cup A, \quad \mathscr{S}(e) \cap A=\emptyset
$$

and so $|\mathscr{S}(f)|=3 k$. Thus, since $\varphi$ is injective,

$$
|\mathscr{P}(f) \varphi|=3 k \text {. }
$$


But $A \varphi \subseteq \mathscr{S}(f) \varphi$; also $\mathscr{S}(f) \varphi$ is left closed since $\mathscr{S}(f) \varphi=\mathscr{S}(f 0)$, by (2). Hence $\overline{A \varphi} \subseteq \mathscr{S}(f) \varphi$. Thus, from (3),

$$
|\overline{A \varphi}| \leqslant 3 k
$$

Let $u, v \in X \cup X^{-1}$ be the first and last letters, respectively, of $x \varphi$. Then, for all $n \in \mathbf{N}, u$ and $v$ are the first and last letters, respectively, of $(x \varphi)^{n}$. Consider the elements listed below:

$$
\begin{aligned}
& (x \varphi)^{r+1},(x \varphi)^{r+2}, \ldots,(x \varphi)^{r+2 k} \\
& (x \varphi)^{r+1} u,(x \varphi)^{r+2} u, \ldots,(x \varphi)^{r+2 k-1} u .
\end{aligned}
$$

Let $p, q \in \mathbf{N}$. If $p \neq q$ then $(x \varphi)^{p} \neq(x \varphi)^{q}$ and $(x \varphi)^{p} u \neq(x \varphi)^{q} u$. Now suppose that $(x \varphi)^{p}=(x \varphi)^{q} u$. Then $p \neq q$, since $u \neq 1$, and so $l\left((x \varphi)^{p-q}\right)>1$. But $(x \varphi)^{p-q}=u$ and $l(u)=1$, which is a contradiction. Thus the $4 k-1$ elements in the list (5) are distinct. Furthermore, for all integers $p$ such that $r+1 \leqslant p \leqslant r+2 k-1,(\mathrm{x} \varphi)^{p} u$ is an initial segment of $(x \varphi)^{p+1}$ if $u \neq v^{-1}$ and is an initial segment of $(x \varphi)^{p}$ if $u=v^{-1}$. It follows that all the elements in the list (5) lie in $\overline{A \varphi}$. Hence $|\overline{A \varphi}| \geqslant 4 k-1$. But this contradicts (4), since $k>1$. Consequently, $l(x \varphi)=1$ for all $x \in X \cup X^{-1}$; that is, $\left(X \cup X^{-1}\right) \varphi \subseteq X \cup X^{-1}$.

Suppose that $\left(X \cup X^{-1}\right) \varphi \neq X \cup X^{-1}$. Then there exists $y \in X \cup X^{-1}$ such that $y \varphi^{-1} \notin X \cup X^{-1}$. Hence

$$
l\left(y \varphi^{-1}\right)>1
$$

Now $\theta^{-1}$ is an isomorphism from $N$ to $M$. By analogy with (2), there exists $\psi \in$ aut $G$ such that

$$
(\forall b \in N), \quad b \theta^{-1}=(\mathscr{S}(b) \psi, b \pi \psi)
$$

Thus, for all $a \in M, a \pi=(a \theta) 0^{-1} \pi=(a \theta) \pi \psi$ and so $a \pi \psi^{-1}=a \theta \pi=a \pi \varphi$. Since $M \pi=G$ this implies that $\psi^{-1}=\varphi$. Hence $\psi=\varphi^{-1}$. The same argument as before, with $N, M, \theta^{-1}, \varphi^{-1}$ replacing $M, N, \theta, \varphi$ respectively, now shows that (6) leads to a contradiction. Hence $\left(X \cup X^{-1}\right) \varphi=X \cup X^{-1}$; that is, $\varphi$ is special.

Leмma 3. Let $\varphi \in$ aut $^{*} G$. Then there exists an automorphism $\propto$ of $S$ such that

$$
(\forall a \in S), \quad a \alpha=(\mathscr{S}(a) \varphi, a \pi \varphi)
$$

Proof. We first note that, for all $g \in G, \overline{g \phi} \sim \bar{g} \phi$ and so, for all $a \in S, \mathscr{f}(a) \phi$ is left closed. Hence we can define a mapping $\alpha: S \rightarrow S$ by

$$
(\forall a \in S), a x=(\mathscr{P}(a) \phi, a \pi \phi) .
$$


Similarly, we can define $x^{\prime}: S \rightarrow S$ by

$$
(\forall a \in S), \quad a \alpha^{\prime}=\left(\mathscr{P}(a) \phi^{-1}, a \pi \phi^{-1}\right)
$$

Then $x^{\prime} x=\imath=\alpha \alpha^{\prime}$, where $\iota$ is the identity mapping on $S$. Hence $\alpha$ is bijective. Moreover, $\alpha$ is a homomorphism, since $\phi$ is a homomorphism. Thus $\alpha \in$ aut $S$.

Remark. From Lemma 2 (with $M=N=S$ ) and Lemma 3 we can recover O'Carroll's theorem linking aut $S$ and aut* $G$.

We now come to the second main result.

THEOREM 2. Let $S$ be a free inverse semigroup, let $M$ and $N$ be ideals of $S$ and let $\theta: M \rightarrow N$ be an isomorphism. Then there exists a unique automorphism a of $S$ such that $\left.\alpha\right|_{M}=0$.

Proof. Let $S=\mathscr{F}_{X_{X}}$, as before. By Lemma 2, there exists $\varphi \in$ aut* $G$ such that

$$
(\forall a \in M), \quad a \theta=(\mathscr{P}(a) \varphi, a \pi \varphi)
$$

Hence, by Lemma 3, there exists $\alpha \in$ aut $S$ such that

$$
(\forall a \in S), \quad a \alpha=(\mathscr{S}(a) \varphi, a \pi \varphi) .
$$

Thus $\left.\alpha\right|_{M}=0$.

Now suppose that $\beta \in$ aut $S$ is such that $\left.\beta\right|_{M}=0$. Then, by Lemma 2 (with $M=N=S$ ), there exists $\psi \in$ aut $^{*} G$ such that

$$
(\forall a \in S), \quad a \beta=(\mathscr{P}(a) \psi, a \pi \psi)
$$

Hence, since $a x=a \beta$ for all $a \in M$, we have that

$$
(\forall a \in M), \quad a \pi \varphi=a \pi \psi .
$$

But $M \pi=G$. Consequently $\phi=\psi$ and so $\alpha=\beta$.

By specializing to the case $M=N$ we readily obtain the following corollary concerning aut $M$.

COROLlary. Let $M$ be an ideal of a free incerse semigroup of $S$. Then

$$
\text { aut } M \cong\{\alpha \in \text { aut } S: M \alpha=M\} \text {. }
$$

Thus. since aut $S \cong \operatorname{aut}^{*} G$, we see that aut $M$ is isomorphic to a subgroup of 
aut* $G$. In particular, if $S$ has finite rank then aut $M$ is finite.

As an application of the corollary above, consider the following sequence of ideals of $S$. For each $n \in \mathbf{N}$ let us write

$$
S_{n}=\{a \in S:|\mathscr{S}(a)| \geqslant n+1\} .
$$

It is almost immediate that each $S_{n}$ is an ideal of $S$ and that

$$
S=S_{1} \supset S_{2} \supset S_{3} \supset \ldots
$$

Now, for all $n \in \mathbf{N}$ and all $\alpha \in$ aut $S$, we have that $S_{n} \alpha=S_{n}$, as can easily be verified with the help of (P3). Hence, from the corollary,

$$
(\forall n \in \mathbf{N}), \quad \text { aut } S_{n} \cong \text { aut } S \text {. }
$$

On the other hand, since the number of elements in a maximal $\mathscr{J}$-class of $S_{k}$ is $(k+1)^{2}$ it follows that

$$
(\forall m, n \in \mathbf{N}), \quad S_{m} \cong S_{n} \Leftrightarrow m=n
$$

In the special case where $S$ has rank 1 the ideals $S_{n}(n \in \mathbf{N})$ are the only ideals of $S$ and hence all ideals have the same automorphism group. namely the group of order 2. It will be shown later that if $S$ has finite rank greater than 1 then $S$ possesses a principal ideal whose automorphism group is trivial.

Next we give a method for testing whether two principal ideals of a free inverse semigroup are isomorphic.

TheOREM 3. Let $S=\mathscr{F} \mathscr{I}_{X}$, let $G=\mathscr{F} \mathscr{G}_{X}$ and let $a, b \in S$. The'n $S a S \cong S b S$ if and only if there exist $\varphi \in$ aut* $G$ and $g \in \mathscr{S}(b)$ such that $\mathscr{S}(a) \varphi=g^{-1} \mathscr{S}(b)$.

ProOf. Suppose first that there exists an isomorphism 0:SaS $\rightarrow$ SbS. Then, by Lemma 2, there exists $\varphi \in$ aut* $G$ such that

$$
(\forall c \in S a S), \quad c \theta=(\mathscr{S}(c) \varphi, c \pi \varphi) .
$$

In particular, $a \theta=(\mathscr{P}(a) \varphi, a \pi \varphi)$ and so $\mathscr{P}(a \theta)=\mathscr{S}(a) \varphi$. But $J_{a}$ and $J_{b}$ are the greatest $\mathscr{J}$-classes of $S a S$ and $S b S$ respectively and so $(a(\theta, b) \in \mathscr{J}$. Thus, by (P5), there exists $g \in \mathscr{S}(b)$ such that $\mathscr{S}(a \theta)=g^{-1} \mathscr{S}(b)$. Consequently, $\mathscr{S}(a) \varphi=g^{-1} \mathscr{S}(b)$.

Conversely, suppose that there exist $\varphi \in$ aut* $G$ and $y \in \mathscr{P}(b)$ such that $\mathscr{S}(a) \varphi=g^{-1} \mathscr{S}(b)$. By Lemma 3, there exists $\alpha \in$ aut $S$ such that

$$
(\forall a \in S), \quad a \alpha=(\mathscr{S}(a) \varphi, a \pi \varphi) .
$$

Now $b=(\mathscr{S}(b), b \pi)$ and so, since there exists $g \in \mathscr{P}(b)$ such that $g^{-1} \mathscr{S}(b)=\mathscr{P}(a) \varphi=\mathscr{P}(a \alpha)$, it follows from (P5) that $(b, a \alpha) \in \mathscr{J}$. Thus $(S a S) \alpha=S(a \alpha) S=S b S$; hence $S a S \cong S b S$. 
The result can be expressed in a simple form making use of the author's concept of a 'word-tree' (Munn (1974)). Each $\mathscr{f}$-class of $S=\mathscr{F}_{\mathscr{I}_{X}}$ corresponds to an unrooted word-tree and two principal ideals of $S$ are isomorphic if and only if the word-trees corresponding to their generating $\mathscr{f}$-classes are obtainable from each other by reversing the orientation of those edges labelled by elements of some subset of $X$ and then relabelling all the edges by applying a permutation to $X$.

The argument used in the first part of the proof of Theorem 3 enables us to show that a free inverse semigroup of finite rank greater than 1 has a principal ideal whose automorphism group is trivial. Let $S=\mathscr{F} \mathscr{I}_{X}$ and $G=\mathscr{F} \mathscr{G}_{X}$, where $2 \leqslant|\mathbf{X}|=n \in \mathbf{N}$, and let the elements of $X$ be $x_{1}, x_{2}, \ldots, x_{n}$. Take

$$
u=\left(\bar{x}_{1} \cup \overline{x_{2}^{2}} \cup \overline{x_{3}^{3}} \cup \ldots \cup \overline{x_{n}^{n}}, 1\right)
$$

and let $\theta \in$ aut $S a S$. Then there exist $\varphi \in$ aut ${ }^{*} G$ and $g \in \mathscr{S}(a)$ such that

(i) $(\forall c \in S(i S), \quad c()=\mid \mathscr{S}(c) \varphi, c \pi \varphi)$

and

(ii) $\mathscr{P}(a) \varphi=g^{-1} \mathscr{F}(a)$.

The elements of $\mathscr{S}(a) \varphi$ of length 1 are $x_{1} \varphi, x_{2} \varphi, \ldots, x_{n} \varphi$. Suppose that $g=x_{i}^{r}$, where $1 \leqslant i \leqslant n$ and $1 \leqslant r \leqslant i$. If $r \neq i$ then $g^{-1} \mathscr{S}(a)$ has precisely two elements of length 1 , namely $x_{i}^{-1}$ and $x_{i}$, while if $r=i$, it has precisely one of length 1 , namely $x_{i}^{-1}$. In either case we obtain a contradiction from (ii). Consequently, $y=1$. Hence, since $\varphi$ is length-preserving, it follows from (ii) that $\varphi$ is the identity automorphism of $G$. Thus, from (i), $\theta$ is the identity automorphism of $S a S$.

Theorem 3 provides a solution to a problem suggested by Reilly (1976). A second problem, also posed by Reilly in the same paper and related to the first, can be solved by similar techniques. We state the result as

THeOREM 4. Let $S=\mathscr{F} \mathscr{I}_{X}$ and let $a, b \in S$. Then $\left\langle J_{a}\right\rangle \cong\left\langle J_{b}\right\rangle$ if and only if there exists an isomorphism

$$
\varphi:\left\langle J_{a}\right\rangle \pi \rightarrow\left\langle J_{b}\right\rangle \pi
$$

and an element $g$ in $\mathscr{S}(b)$ such that $\mathscr{S}(a) \varphi=g^{-1} \mathscr{S}(b)$. (Note that $\mathscr{S}(a) \subseteq\left\langle J_{a}\right\rangle \pi$, since $\left.\mathscr{S}(a)=R_{a} \pi, b y(\mathrm{P} 2).\right)$

Proof. Write $T=\left\langle J_{a}\right\rangle$ and $U=\left\langle J_{b}\right\rangle$. Since $J_{a}^{-1}=J_{a}$ we see that $T$ is an inverse subsemigroup of $S$. Similarly, $U$ is an inverse subsemigroup of $S$.

Suppose that there exists an isomorphism $\theta: T \rightarrow U$. Since $J_{a}$ and $J_{b}$ are, respectively, the greatest $\not$-classes of $T$ and $U$, it follows that $J_{a} \theta=J_{b}$. Hence, in 
particular, $R_{a \theta} \subseteq U$. Thus, by Lemma 1 , there exists an isomorphism $\varphi: T \pi \rightarrow U \pi$ such that

$$
a \theta=(\mathscr{S}(a) \varphi, a \pi \varphi)
$$

Hence $\mathscr{S}(a \theta)=\mathscr{S}(a) \varphi$. But, by (P5), since $(a \theta, b) \in \mathscr{J}$ there exists $g \in \mathscr{P}(b)$ such that $\mathscr{P}(a 0)=g^{-1} \mathscr{S}(b)$. Thus $\mathscr{S}(a) \varphi=g^{-1} \mathscr{S}(b)$.

Conversely, suppose that there exists an isomorphism $\varphi: T \pi \rightarrow U \pi$ and an element $g$ in $\mathscr{S}(b)$ such that $\mathscr{P}(a) \varphi=g^{-1} \mathscr{P}(b)$. First, we note that, for all $h \in \mathscr{P}(a)$,

$$
\begin{aligned}
\left(h^{-1} \mathscr{P}(a)\right) \varphi & =(h \varphi)^{-1} \mathscr{P}(a) \varphi \\
& =(h \varphi)^{-1} g^{-1} \mathscr{S}(b) \\
& =(g(h \varphi))^{-1} \mathscr{S}(b)
\end{aligned}
$$

furthermore, $g(h \varphi) \in \mathscr{S}(b)$, since $h \varphi \in \mathscr{S}(a) \varphi=g^{-1} \mathscr{P}(b)$. Combining this with (P5) we see that, for all $h \in \mathscr{P}(a)$ and all $k \in h^{-1} \mathscr{S}(a)$,

$$
\left(\left(h^{-1} \mathscr{P}(a)\right) \varphi, k \varphi\right) \in J_{b}
$$

Now consider an element $c \in T$. By the definition of $T$ there exist $c_{1}, c_{2}, \ldots, c_{r} \in J_{a}$ such that $c=c_{1} c_{2} \ldots c_{r}$. But $\mathscr{S}\left(c_{i}\right)=h_{i}^{-1} \mathscr{S}(a)$ for some $h_{i}$ in $\mathscr{S}(a)$, by (P5), and so, by (7), $\left(\mathscr{S}\left(c_{i}\right) \varphi, c_{i} \pi \varphi\right) \in J_{b}(i=1,2, \ldots, r)$. Thus

$$
(\mathscr{P}(c) \varphi, c \pi \varphi)=\left(\mathscr{P}\left(c_{1}\right) \varphi, c_{1} \pi \varphi\right) \ldots\left(\mathscr{P}\left(c_{r}\right) \varphi, c_{r} \pi \varphi\right) \in\left\langle J_{b}\right\rangle=U .
$$

We can therefore define a mapping $\theta: T \rightarrow U$ by the rule that

$$
(\forall c \in T), \quad c \theta=(\mathscr{P}(c) \varphi, c \pi \varphi) \text {. }
$$

Clearly $\theta$ is a homomorphism; moreover, since $\varphi$ has an inverse it follows that $\theta$ is injective. We show that $\theta$ is surjective. It will suffice to prove that $J_{b} \subseteq J_{a} \theta$.

Let $d \in J_{b}$. By (P5), there exists $k \in \mathscr{S}(b)$ such that $\mathscr{P}(d)=k^{-1} \mathscr{S}(b)$. But $\mathscr{P}(b)=g(\mathscr{S}(a) \varphi)$, by hypothesis. Hence $k=g(h \varphi)$ for some $h \in \mathscr{P}(a)$ and so

$$
k^{-1} \mathscr{P}(b)=(h \varphi)^{-1} g^{-1}[g(\mathscr{S}(a) \varphi)]
$$

that is,

$$
\mathscr{S}(d)=\left(h^{-1} \mathscr{S}(a)\right) \varphi .
$$

Also $d \pi \in \mathscr{S}(d)$ and so, by $(9), d \pi=m \varphi$ for some $m \in h^{-1} \mathscr{S}(a)$. But $\left(h^{-1} \mathscr{P}(a), m\right) \in J_{a}$, by (P5). Hence, by (9) and (8),

$$
d=\left(\left(h^{-1} \mathscr{P}(a)\right) \varphi, m \varphi\right)=\left(h^{-1} \mathscr{f}(a), m\right) \theta \in J_{a} \theta
$$

Thus $J_{b} \subseteq J_{a} \theta$, as required.

The mapping $\theta$ is therefore an isomorphism and the proof is complete. 
To conclude, we give an example to illustrate Theorems 3 and 4. Let $X=\{x, y, z\}$, let $S=\mathscr{F} \mathscr{I}_{X}$ and let $a, b \in S$ be defined as follows:

$$
a=(\{1, x, x y, x y x\}, 1), \quad b=\left(\left\{1, x, z^{-1}, z^{-1} x\right\}, 1\right) .
$$

Let $G=\mathscr{F} \mathscr{G}_{X}$. Then it can be verified, by exhaustion of cases, that there does not exist $(\varphi, g)$ in aut ${ }^{*} G \times \mathscr{P}(b)$ such that $\mathscr{S}(a) \varphi=g^{-1} \mathscr{S}(b)$. Thus, by Theorem 3, $S a S \nsubseteq S b S$.

Now $\left\langle J_{a}\right\rangle \pi$ and $\left\langle J_{b}\right\rangle \pi$ are, respectively, the free groups on $\{x, y\}$ and $\{x, z\}$. Let $\varphi:\left\langle J_{a}\right\rangle \pi \rightarrow\left\langle J_{b}\right\rangle \pi$ be the isomorphism defined by

$$
x \varphi=x, \quad y \varphi=x^{-1} z .
$$

Then

$$
\mathscr{P}(a) \varphi=\{1, x, z, z x\}=z \mathscr{S}(b)
$$

and $z^{-1} \in \mathscr{S}(b)$. Hence, by Theorem $4,\left\langle J_{a}\right\rangle \cong\left\langle J_{b}\right\rangle$.

\section{Acknowledgements}

The main part of this work was carried out while I held a visiting appointment at Monash University. I wish to express my gratitude to the university authorities for their support and my thanks to the members of the Mathematics Department for many helpful and stimulating discussions.

\section{References}

J. M. Howie (1976), An introduction to semigroup theory (Academic Press, London).

W. D. Munn (1961). 'A class of irreducible matrix representations of an arbitrary inverse semigroup', Proc. Glasyow Math. Assoc. 5. 4148.

W. D. Munn (1974), 'Free inverse semigroups', Proc, London Math. Soc. (3) 29, 385-404.

W. D. Munn (to appear) 'An embedding theorem for free inverse semigroups', Glasgow Math. $J$.

L. O'Carroll (1974), 'A note on free inverse semigroups', Proc. Edinhurgh Math. Soc. (2) 19, 17-23.

N. R. Reilly (1972), 'Free generators in free inverse semigroups', Bull. Austral. Math. Soc. 7, 407-424.

N. R. Reilly (1973), 'Free generators in free inverse semigroups: corrigenda', Bull. Austral. Math. Soc.9, 479.

N. R. Reilly (1976), 'Free inverse semigroups', Algebraic theory of semigroups, pp. 479-508 (Colloquia Mathematica Societatis János Bolyai 20).

H. E. Scheiblich (1972), 'Free inverse semigroups', Semigroup Forum 4, 351-359.

H. E. Scheiblich (1973), 'Free inverse semigroups', Proc. Amer. Math. Soc. 38, 1-7.

Department of Mathematics

University of Glasgow

Glasgow G12 8QW

Scotland 